\title{
Alteration of MR-DWI/ADC before and 24h after induction of chemotherapy in patients with lung cancer
}

\author{
O Sedlaczek*, C Wiedemann, C Gruellich, U Klingmüller, HU Kauczor, HP Schlemmer \\ From International Cancer Imaging Society (ICIS) 14th Annual Teaching Course \\ Heidelberg, Germany. 9-11 October 2014
}

\section{Background}

Diffusion weighted MRI (MR-DWI) is frequently used in oncologic therapy monitoring as it is known to predict therapy response much earlier, than measurements of tumour size alone. In several studies the outcome of chemotherapeutic treatment of brain, soft tissue, bone, breast and prostate tumours was predicted by changes in MR-DWI. Usually, follow-up studies 2 to 4 weeks after induction of chemotherapy show an increase in the apparent diffusion coefficient (ADC), predicting size changes at the end the treatment cycle. DWI measurements in patients with NSCLC ultra early after starting chemotherapy are missing.

\section{Patients \& methods}

23 patients with lung cancer (aged $63.6 \pm 7.2$ years) underwent serial MRI before and $24 \mathrm{~h}$ after starting platin-based chemotherapeutical regimens. The MRI protocol contained a DWI sequence with $6 \mathrm{~b}$-values ranging from 0 to 800. Online calculated trace images and the apparent diffusion coefficient (ADC) were used for response evaluation. In 19 patients both clinical information and RECIST evaluations at the end of the second chemotherapy cycle were available.

\section{Results}

18 out of 23 patients showed a decrease in ADC maps $24 \mathrm{~h}$ after starting treatment.

In three of the 19 patients with available follow-up data, no initial ADC reduction was observed. In all of these patients a progressive disease was observed by the time of completing the second therapy cycle. In 14 patients, initial

* Correspondence: o.sedlaczek@dkfz.de

Department of Radiology, German Cancer Research Center, Heidelberg, Germany 